\title{
Conservation in first introns is positively associated with the number of exons within genes and the presence of regulatory epigenetic signals
}

\author{
Seung Gu Park², Sridhar Hannenhalli ${ }^{i^{*}}$ and Sun Shim $\mathrm{Choi}^{2^{*}}$
}

\begin{abstract}
Background: Genomes of higher eukaryotes have surprisingly long first introns and in some cases, the first introns have been shown to have higher conservation relative to other introns. However, the functional relevance of conserved regions in the first introns is poorly understood. Leveraging the recent ENCODE data, here we assess potential regulatory roles of conserved regions in the first intron of human genes.

Results: We first show that relative to other downstream introns, the first introns are enriched for blocks of highly conserved sequences. We also found that the first introns are enriched for several chromatin marks indicative of active regulatory regions and this enrichment of regulatory marks is correlated with enrichment of conserved blocks in the first intron; the enrichments of conservation and regulatory marks in first intron are not entirely explained by a general, albeit variable, bias for certain marks toward the $5^{\prime}$ end of introns. Interestingly, conservation as well as proportions of active regulatory chromatin marks in the first intron of a gene correlates positively with the numbers of exons in the gene but the correlation is significantly weakened in second introns and negligible beyond the second intron. The first intron conservation is also positively correlated with the gene's expression level in several human tissues. Finally, a gene-wise analysis shows significant enrichments of active chromatin marks in conserved regions of first introns, relative to the conserved regions in other introns of the same gene.
\end{abstract}

Conclusions: Taken together, our analyses strongly suggest that first introns are enriched for active transcriptional regulatory signals under purifying selection.

\section{Background}

Recent complete sequencing projects have confirmed that almost all eukaryotes have introns [1-6]. Different species harbor dramatically different density and length of introns, ranging from a few bps to hundreds kbps $[5,7,8]$. Generally speaking, genes in higher eukaryotes such as mammals have a greater number of introns than those of lower eukaryotes such as yeast, Drosophila, and $C$. elegans $[5,7,8]$. These differences may partly be explained by the differences in modes of intron removal between lower and higher eukaryotes [9], as well as differences in selective pressure. A substantial fraction of introns in the

\footnotetext{
* Correspondence: sridhar@umiacs.umd.edu; schoi@kangwon.ac.kr 'Department of Cell Biology and Molecular Genetics, Center for Bioinformatics and Computational Biology, University of Maryland, College Park, Maryland, MD 20742, USA

${ }^{2}$ Department of Medical Biotechnology, College of Biomedical Science, and Institute of Bioscience \& Biotechnology, Kangwon National University,
} Chuncheon 200-701, South Korea human genome have likely originated early in eukaryotic evolution, and dynamic evolutionary changes such as intron gain and loss have structured the eukaryotic introns since [10-14]. Noticeably, the number of genes is relatively stable across organisms from C. elegans $(\sim 19,000)$ and Drosophila $(\sim 14,000)$ to humans $(\sim 25,000)$, while the fraction of non-coding DNA including introns greatly varies up to several folds $[15,16]$, some of which is likely to underlie species-specific adaptations $[17,18]$.

The mere existence of introns in the eukaryotic genome is intriguing, given the cost of transcription and the cost of maintaining a splicing regulatory system that ultimately eliminates the introns from the functional product of the gene. In particular, whether introns have evolved under selective constraints, and the extent thereof, are not entirely clear; while some studies suggest that introns evolve largely free from selective constraints [19-22], others imply that intron sequences are subject to considerable 
levels of evolutionary constraints [22-25]. Using intronic multispecies conserved sequences (MCSs), Sironi et al. [26] showed that the MCS density steadily increase with intron length with MCSs occupying up to $10 \%$ of total size in long introns, and also that MCSs are enriched in genes involved in development and transcription. Based on 225 intron fragments in D. melanogaster and D. simulans, Haddrill et al. [27] demonstrated that a substantial portion of intronic sites is likely to be evolving under considerable selective constraint and this tendency increases with intron length. Furthermore, Vinogradov [28] showed that the length of conserved intronic sequence in the human genome is greater in proteins with the larger numbers of functional domains. Even though several reports have shown an enrichment of conserved sequences in introns, in various species [23-25,28,29], these claims are not without controversies. In one instance, it was shown that intronic sequences evolve faster than fourfold degenerate sites when splicing regulatory sequences were excluded [22]. These discrepancies can be partly ascribed to biases in the data sets with different ranges of lengths of introns studied [27]. Besides their obvious role in isoform regulation, introns have also been shown to harbor regulatory signals and noncoding genes [30-33]. Thus, overall, it is highly likely that portions of intronic sequences are evolving under selective constraints consistent with their functional importance.

The 5'-most "first" intron differs from the other introns in several aspects in terms of processing, epigenomic marks, length and evolution. First, in terms of processing, despite an overall 5'-to-3' trend in splicing during transcription, the first intron is removed (on average) somewhat later [34]. Second, the 5' end of the first intron displays a specific epigenomic context being enriched for two activating histone modifications H3K4me3 and H3K9ac [35]. Finally, the "first" introns have a special status, as these are typically the longest among all introns and appear to be most selectively constrained. More conserved blocks were found in first introns relative to other introns in several species [36]. Consistently, some studies have shown that intron divergence has a significant negative correlation with the length of first introns in Drosophila [27,37]. Zheng et al. [38] have also reported that, in Tetrahymena, the most conserved introns are found closer to the 5 ' end of genes. Furthermore, introns harboring regulatory elements tend to be the first introns [38-40], and, in fact, the frequencies of certain regulatory motifs are greater in first introns [41]. Overall, it seems that, among the various possible roles of introns, first introns have especially evolved to harbor regulatory elements.

Previous investigations of potential regulatory role of first intron were mainly performed in Drosophila or plants. The extent to which the previous conclusions hold true in mammals is not known. Moreover, the recent explosion of human epigenomic data via the ENCODE project (Table 1) provides a unique opportunity to investigate regulatory potential of first introns and its correlates thereof in human. Based on human RefSeq gene annotation from UCSC and 46 vertebrate species conservation (including primate and mammal subsets) (Table 1), we first show that blocks of highly conserved sequences are significantly enriched in first introns relative to other introns. Using genome-wide profiles of several epigenomic marks from the ENCODE database, we show that the first introns are also enriched for chromatin marks indicative of active regulatory regions in a manner consistent with conserved blocks. Interestingly, conservation in first intron as well as active chromatin marks of a gene correlate positively with the numbers of exons in the gene. While these correlations also hold true for conservation and active marks in gene's upstream region, they are significantly weaker in second introns and negligible beyond the second intron. In summary, our results strongly suggest that first introns in human are enriched for evolutionarily selected active transcriptional regulatory signals that are likely to be important for regulating complex gene expression patterns of large multidomain genes.

\section{Results}

\section{First introns are the most conserved}

As a proxy for purifying selection, we compared evolutionary conservation across introns grouped by their position in the transcript structure. Conservation in an intron was estimated as the fraction of intron sites that were conserved based on a PhastCons score threshold. Three different multiple alignments (primates, mammals and vertebrates) and different PhastCons score thresholds were used. Our conclusions hold for all choices of alignment and threshold; here we only present the results based on mammalian conservation with PhastCons score threshold of 0.5. The primary focus of our investigation was transcriptional regulatory elements in introns. Therefore, to exclude the possibility of splice site signals biasing the conservation score, especially for short introns, the sequences within $300 \mathrm{bps}$ of the splice junction, which are considered to harbor splicing regulation signals [42], were excluded from our analysis. Based on these criteria, the median conservation per intron was only $2.1 \%$. Introns were grouped by their positions from the 5 ' end of the transcript; for instance, all first introns were in the first group. The fraction of conserved sites was then estimated within each group.

We found that the median conservation in first introns (3.3\%) was significantly higher than all other groups using Wilcoxon rank sum test $(\mathrm{p}<2.2 \mathrm{e}-16)$ (Figure 1$)$. A potential bias in this analysis stems from the fact that shorter transcripts with few introns are more abundant relative to long genes with several introns and will therefore 
Table 1 List of data resources

\begin{tabular}{ll}
\hline Resources & URLs \\
\hline ENCODE & http://www.nature.com/encode/\#/threads \\
UCSC chromosome sequence & http://hgdownload.soe.ucsc.edu/goldenPath/hg19/chromosomes/ \\
$\mathbf{4 6}$ species multiple alignment & http://hgdownload.cse.ucsc.edu/goldenpath/hg19/multiz46way/alignments/ \\
The Genome Reference Consortium & http://www.ncbi.nlm.nih.gov/projects/genome/assembly/grc \\
PhastCons & http://hgdownload.cse.ucsc.edu/goldenPath/hg19/phastCons46way/ \\
$\begin{array}{l}\text { DNasel Hypersensitivity Uniform Peaks from } \\
\text { ENCODE/Analysis }\end{array}$ & http://genome.ucsc.edu/cgi-bin/hgFileUi?db=hg19\&g=wgEncodeAwgDnaseUniform \\
$\begin{array}{l}\text { Transcription Factor ChIP-seq Uniform Peaks from } \\
\text { ENCODE/Analysis }\end{array}$ & http://genome.ucsc.edu/cgi-bin/hgFileUi?db=hg19\&g=wgEncodeAwgTfbsUniform \\
$\begin{array}{l}\text { Histone Modifications by ChIP-seq from } \\
\text { ENCODE/Broad Institute }\end{array}$ & http://genome.ucsc.edu/cgi-bin/hgFileUi?db=hg19\&g=wgEncodeBroadHistone \\
RNA-Seq Atlas & http://medicalgenomics.org/rna_seq_atlas/download \\
\hline
\end{tabular}

dominate the first intron group. To avoid this potential bias we repeated the analysis with different sets of transcripts grouped by number of introns, i.e., transcripts were first segregated based on the number of introns, and the analysis was repeated separately for all the groups, as illustrated in Additional file 1: Figure S1A. As shown in Additional file 1: Figure S1B, a similar trend was observed: first introns are the most conserved.

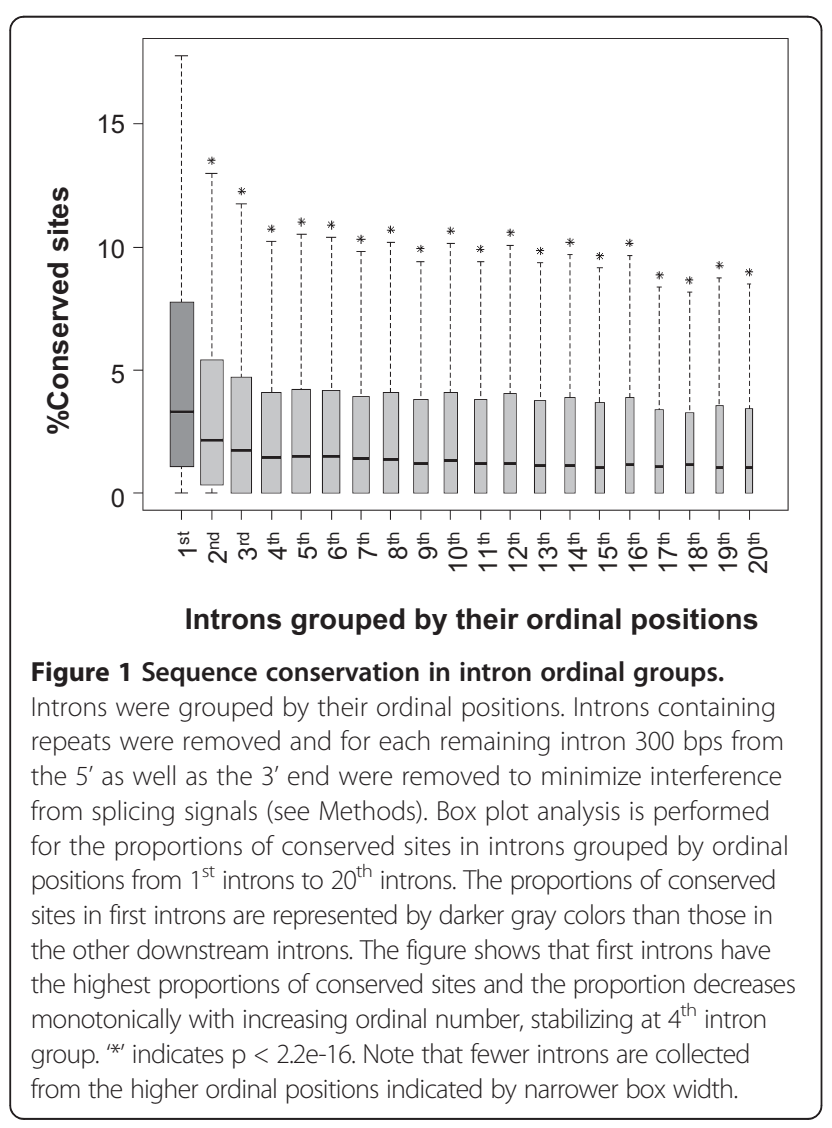

Chromatin signals are the highest in first introns and increase with increasing conservation

Conserved regions in first intron are likely to play a transcriptional regulatory role as suggested by previous studies [43-51]. Several chromatin marks have been shown to associate with transcriptional regulatory regions [52-54]. Next we assessed whether the first introns are enriched for specific regulatory signals, similar to the conservation analysis. We obtained a number of chromatin marks and protein-binding data from ENCODE $[55,56]$ for three cell lines - GM12878, H1-hESC, and K562. The following data were included: DNase I hypersensitivity sites (DHS), Transcription factor binding sites (TFBSs) for 80 TFs in GM12878, 50 TFs in H1-hESC, and 112 TFs in K562, active chromatin marks (e.g., H3K4me1, H3K4me3), a repressive chromatin mark (e.g., H3K9me3), and the insulator protein CTCF binding sites.

Figure 2 shows the regulatory signals in different intron groups. The results in different cell lines were similar and here we show the results based on data from GM12878 while the results based on other cell lines are provided as Supplementary Figures. Our analysis shows a clear enrichment of epigenomic signals in first introns relative to other introns (Figure 2 and Additional file 1: Figure S2A-B). Next we assessed whether enrichment of epigenomic signals in introns correlated with that for conservation. As shown in Figure 3 and Additional file 1: Figure S3A-B, we found this to be generally the case, significant $(\mathrm{p}<0.01)$, the correlations are relatively modest $(\tau \sim 0.2)$, specifically for active chromatin marks, DHS, and TFBS. However, there was weak or no correlation for repressive chromatin marks such as H3K9me3 and CTCF binding sites. These trends are consistent in the two other cell types, hESC and K562 (Additional file 1: Figure S2A-B).

Next we contrasted the above conservation results for first introns with that for proximal promoters of the genes, 


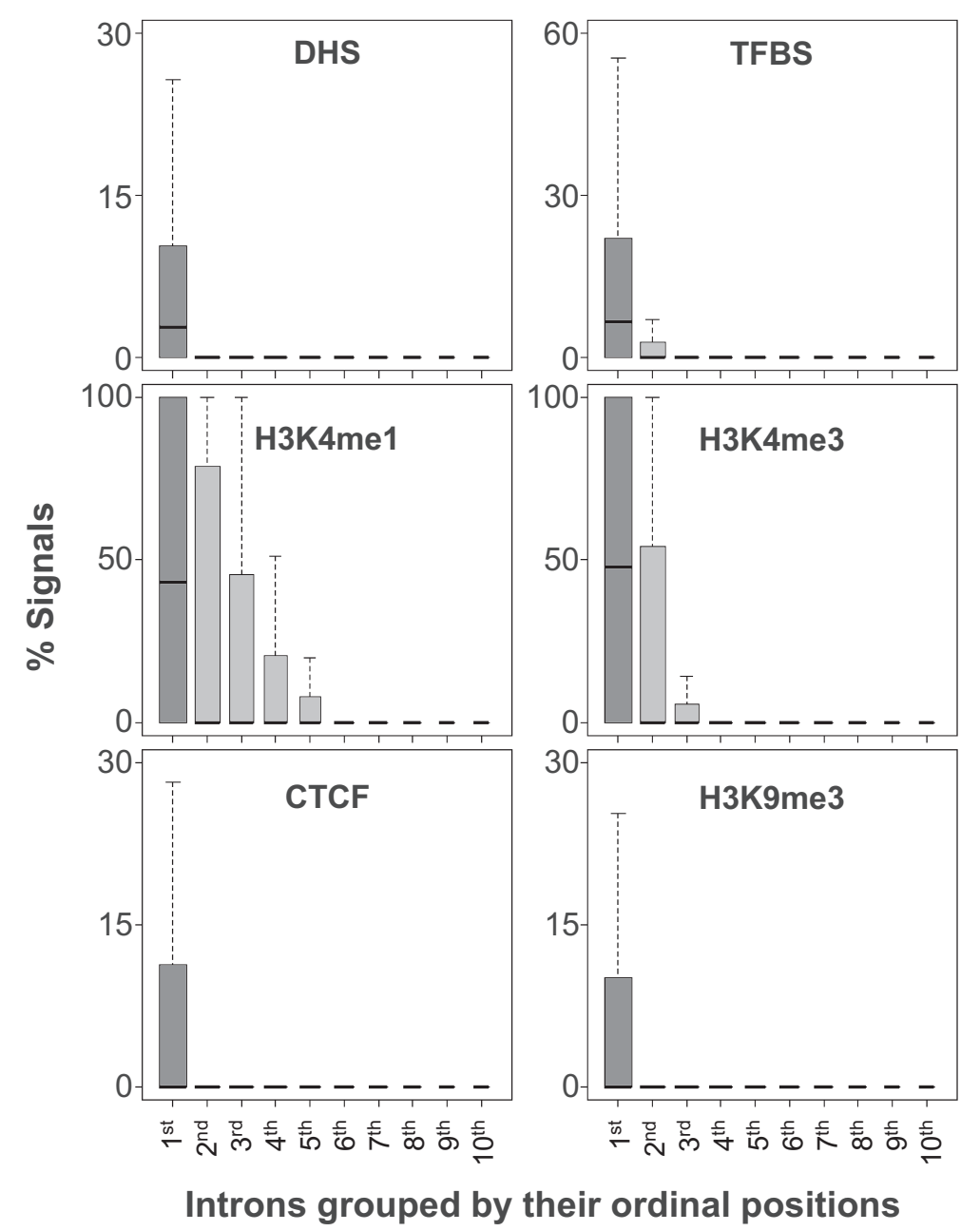

Figure 2 Proportions of regulatory chromatin marks in intron ordinal groups. All the signals are derived from GM12878 cell line. Using the peak values for each signal, box plot analysis is performed for the proportions of the chromatin marks sites in introns from each ordinal group are estimated. Results of the same analyses in the two other cell lines are presented in Additional file 1: Figure S2A, B. The proportions of the peak signals of each chromatin regulatory marks in first introns are represented by darker gray colors than those in the other downstream introns. As shown, the proportions of the regulatory chromatin marks are found to be the highest in first introns compared to the other downstream introns.

known to harbor conserved regulatory signals [57-61]. We found that the correlations between conservation and epigenomic marks also hold for $2 \mathrm{~kb}$ proximal promoter regions of the gene (Additional file 1: Figure S4A-C), which suggests an enrichment of conserved regulatory signals specifically in first introns, akin to proximal promoters.

First intron conservation and regulatory signals positively correlate with the numbers of exons

Given that mammalian first introns can often be very long and thus harbor numerous conserved, potentially regulatory regions, we assessed the relationship between level of conservation and the complexity of gene's expression. From an information theoretic perspective, expression complexity is defined in terms of the number of tissues (or conditions) in which a gene is expressed. Genes that expressed in a very few or most tissues have low complexity whereas the genes expressed in an intermediate number of tissues have the highest expression complexity $[28,62]$. It was shown that expression complexity is positively correlated with both coding and intronic content of the gene $[28,62]$. Therefore we used number of exons as a proxy for gene expression complexity. We then estimated the correlation between the first intron conservation and number of exons for all genes. Interestingly, we found striking positive correlations between conservation in first intron and the numbers of exons (Figure 4). A similar but weak positive trend was found in the case of conservations in second introns but the trend greatly weakened after second intron (Figure 4). We compared the results for first introns with that for the $2 \mathrm{~kb}$ proximal 


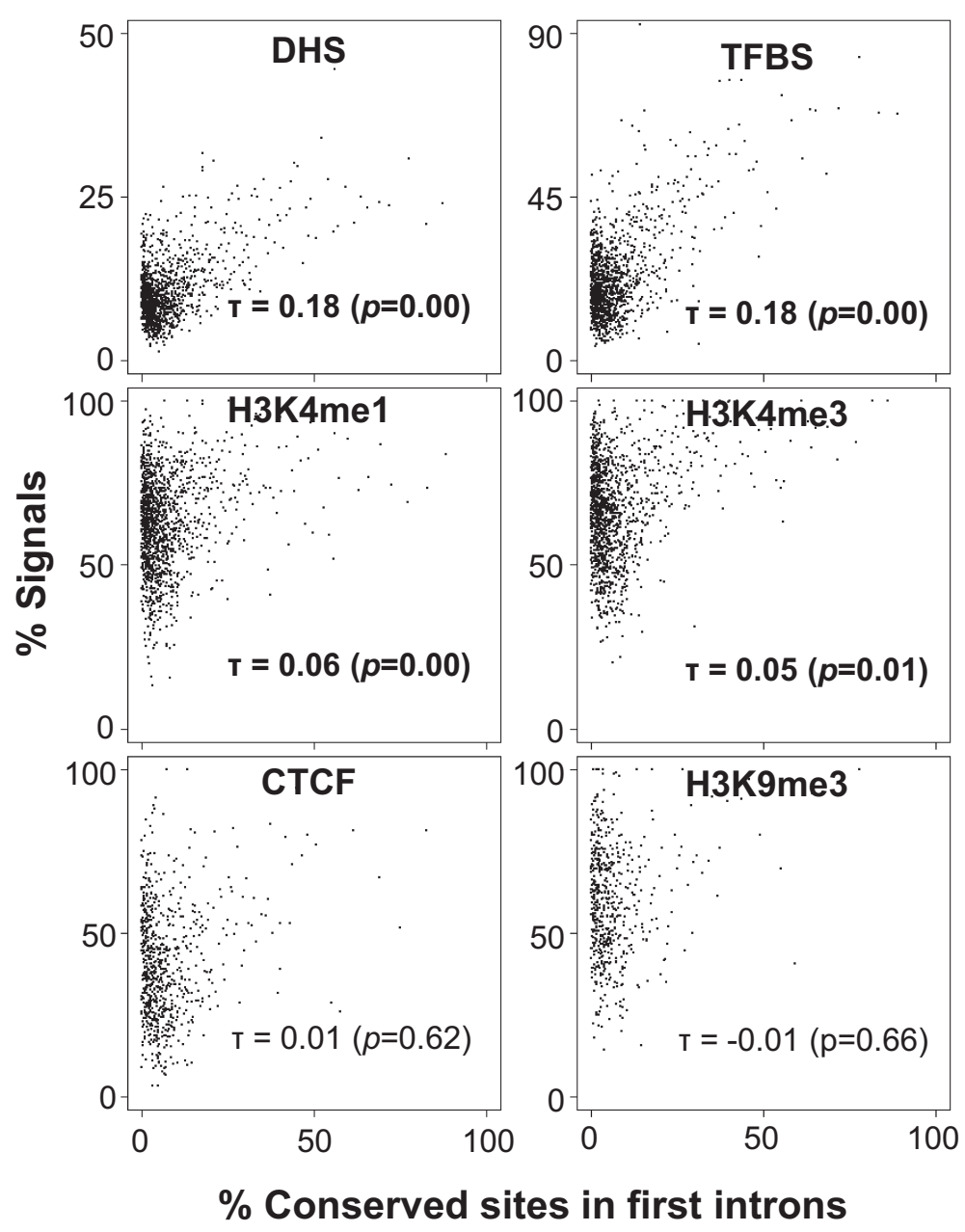

Figure 3 Correlation between regulatory signals and conservation in first introns. Kendall's tau correlation analysis is performed to test how the conservation in first introns is related to density of regulatory marks. For smoothing, introns are binned into groups of 10 genes by conservation and average regulatory signal density is calculated for each bin, and plotted against the average conservation of the group. As in Figure 2, all marks are obtained from GM12878 cell line, and the results from the other two cell lines are provided in Additional file 1: Figure S3A, B. Kendall's tau values and $p$-values are shown; significant $p$-values $(p<0.05)$ are represented by bold font. All active chromatin marks show significant positive correlations between conservation and the proportions of the regulatory marks.

promoter region. As expected, the promoter conservations correlated positively with the numbers of exons (Additional file 1: Figure S5). However, when the same analysis was performed for $2 \mathrm{~kb}$ downstream flanking regions of genes, as shown in Additional file 1: Figure S5, the trend was still significant but very weak or non-existent.

Next we assessed the correlation between the regulatory signals and the number of exons. As shown in Figure 5 and Additional file 1: Figure S6, general regulatory signals (e.g., DHS and TFBS) and active regulatory chromatin marks (e.g., H3K4me1 and H3K4me3) exhibit a significant positive correlation between the regulatory signal proportion and the number of exons, whereas CTCF binding sites and a repressive regulatory chromatin mark, H3K9me3 did not show any such trend. This finding suggests that conservation may have to do with the presence of active regulatory signals rather than of repressive regulatory signals.

\section{Conservation and active epigenomic marks correlate with} level of gene expression

We next investigated whether or not conservation in first introns is related to the level of gene expressions. For this, the degrees of conservation were plotted against the levels of gene expressions in several human tissues. Gene expression data sets were obtained from RNA-seq atlas constructed by [63] from 11 human tissues. Here, we only show a subset of the results from four different tissues including hypothalamus, heart, skeletal muscle, and lung (Figure 6), although the conclusions were the same for all the other tissues (data not shown). Interestingly, conservation is positively correlated with the levels of gene 


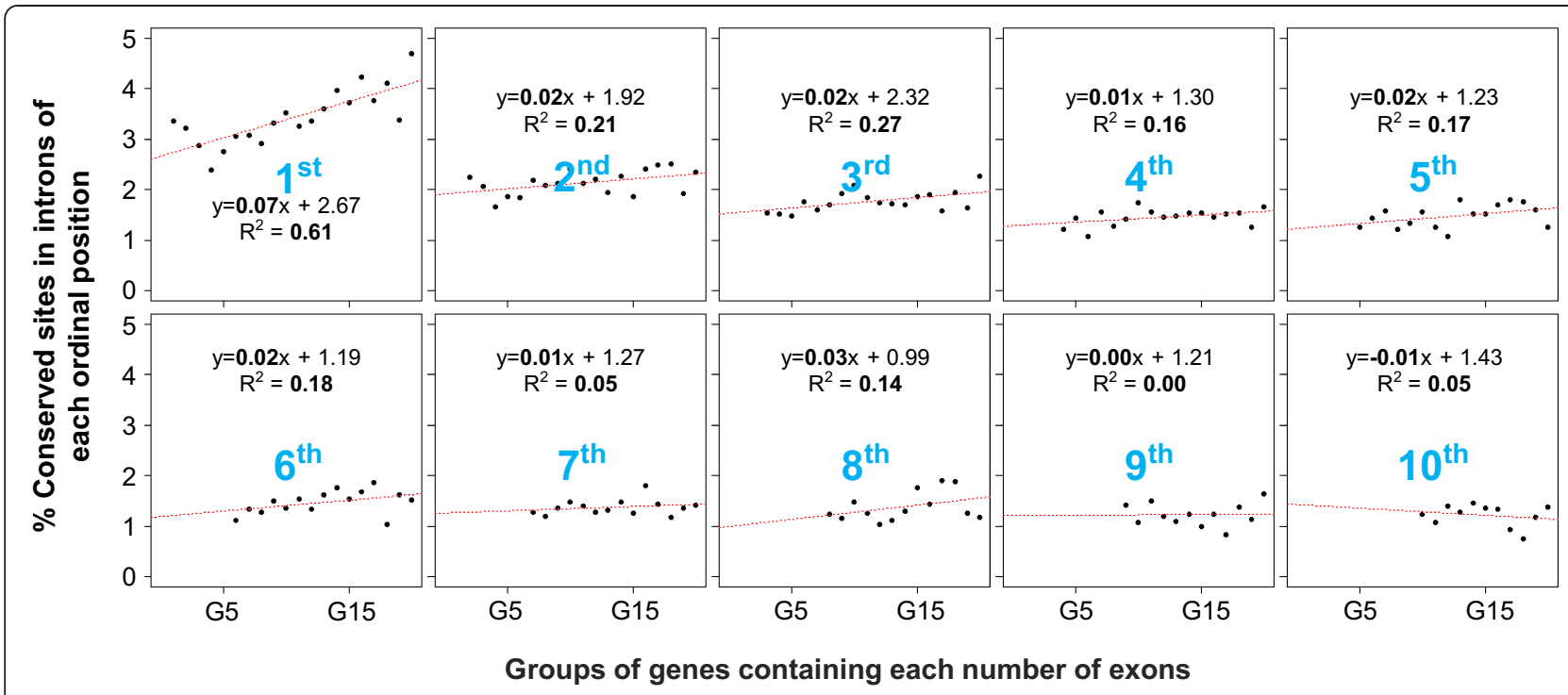

Figure 4 Relationship between intron conservation and the numbers of exons. Linear regression analysis is performed to see the relationship between the degree of conservation in introns from each ordinal position and the numbers of exons within genes. Genes are grouped by the numbers of exons within genes. For example, as shown in the top left box in the figure, genes with two exons are grouped together (named G1), the average degrees of conservations in first introns of the genes in G1 in X-axis is shown on the Y-axis. As for G1, the conservations in first introns in genes with three exons (named G2) and up to genes with twenty-one exons (named G20) are calculated. Likewise, in the box for $2^{\text {nd }}$ introns (shown in blue), genes are grouped as in the first box but now the conservation in second intron is estimated; likewise for introns 3 up to 10. Note that the numbers of dots decreases by one in each subsequent box, because $\mathrm{N}^{\text {th }}(\mathrm{N}>=1)$ introns are non-existent in genes comprising less than $\mathrm{N}$ numbers of exons. Regression equations and R-squared values for each linear regression analysis are shown. The collection of plots suggests that there is strong correlation between first intron conservation and number of exons, specifically for the first intron, and much lesser extent for other introns.

expression in all tissue types. The degrees of conservations in $2 \mathrm{~kb}$ proximal promoter also showed the same positive trends (Figure 6), confirming that the conserved sites in first introns and promoters might have something to do with the presence of active regulatory signals rather than repressive signals, consistent with the results of Figure 3. There was no significant positive correlation between the degrees of conservation in 3' flanking region and expression levels (Figure 6).

Although the analysis thus far was based on all 'transcripts', we repeated the analyses by selecting a single representative transcript per gene, resulting in $\sim 16,000$ transcripts. As shown in Additional file 1: Figure S7A-D, the analyses at the gene level yield qualitatively the same results.

\section{Regulatory signals preferentially occur in conserved regions in first introns - a gene-wise assessment}

Our analysis thus far is based on grouped data. Next we assessed for each gene whether the regulatory signals in the first intron preferentially occur within conserved region. We partitioned each first intron into conserved and nonconserved regions, obtained percent coverage by regulatory signals in each part and calculated the log-odds ratio with 95\% confidence intervals (see Methods) (Figure 7 and Additional file 1: Figure S8). Note that the total numbers of genes in each panel in Figure 7, corresponding to different regulatory marks, are different because of missing data for some genes in each case. As shown in Figure 7, for some of the signals (DHS, TFBS, H3K4me3) there are many more genes with significant enrichment of the signal in conserved regions relative to the genes with significant enrichment in non-conserved regions of the first intron. For some other signals (H3K4me1, H3K9me3) the opposite is true, while for CTCF, there is no clear winner. An enrichment of accessible chromatin and TFBS binding in conserved regions of the first intron is consistent with their role in active gene regulation. We also found that that promoter-specific mark H3K4me3 [54] is also enriched among conserved regions in first introns, while H3K4me1, which is associated with both enhancers and promoters, is not. Also, a lack of enrichment of repressive chromatin mark $\mathrm{H} 3 \mathrm{~K} 9 \mathrm{me} 3$ in the conserved regions is consistent with the result of Figure 3 showing weak or no correlation between the proportions of the repressive chromatin marks in first introns and the degrees of conservation.

\section{Trends in the first introns are independent from those in promoters}

A substantial portion of transcriptional control of a gene is mediated by signals in its proximal promoters. In fact, numerous ChIP-seq data for TFBS from the ENCODE project reveal a distribution of binding sites around the 


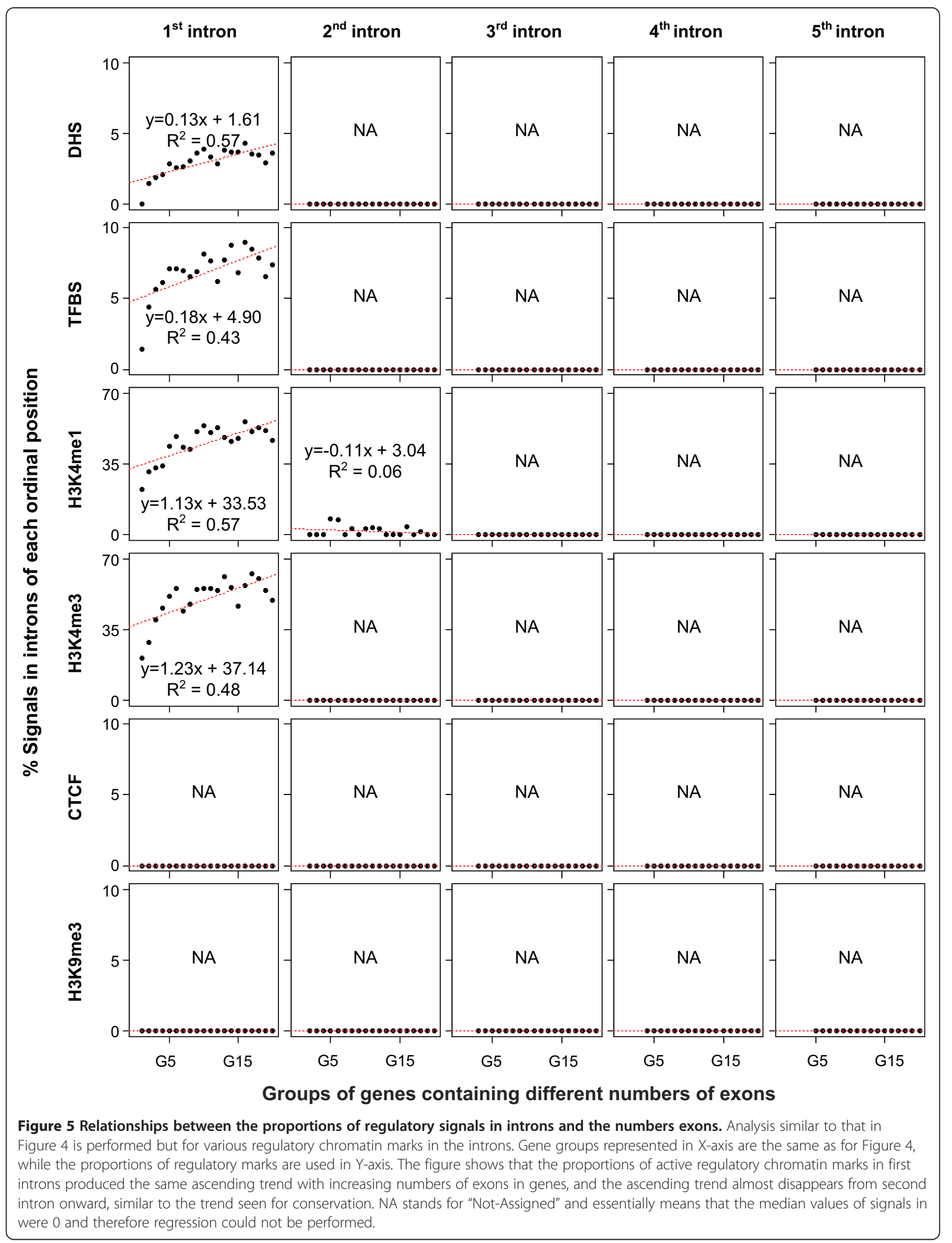




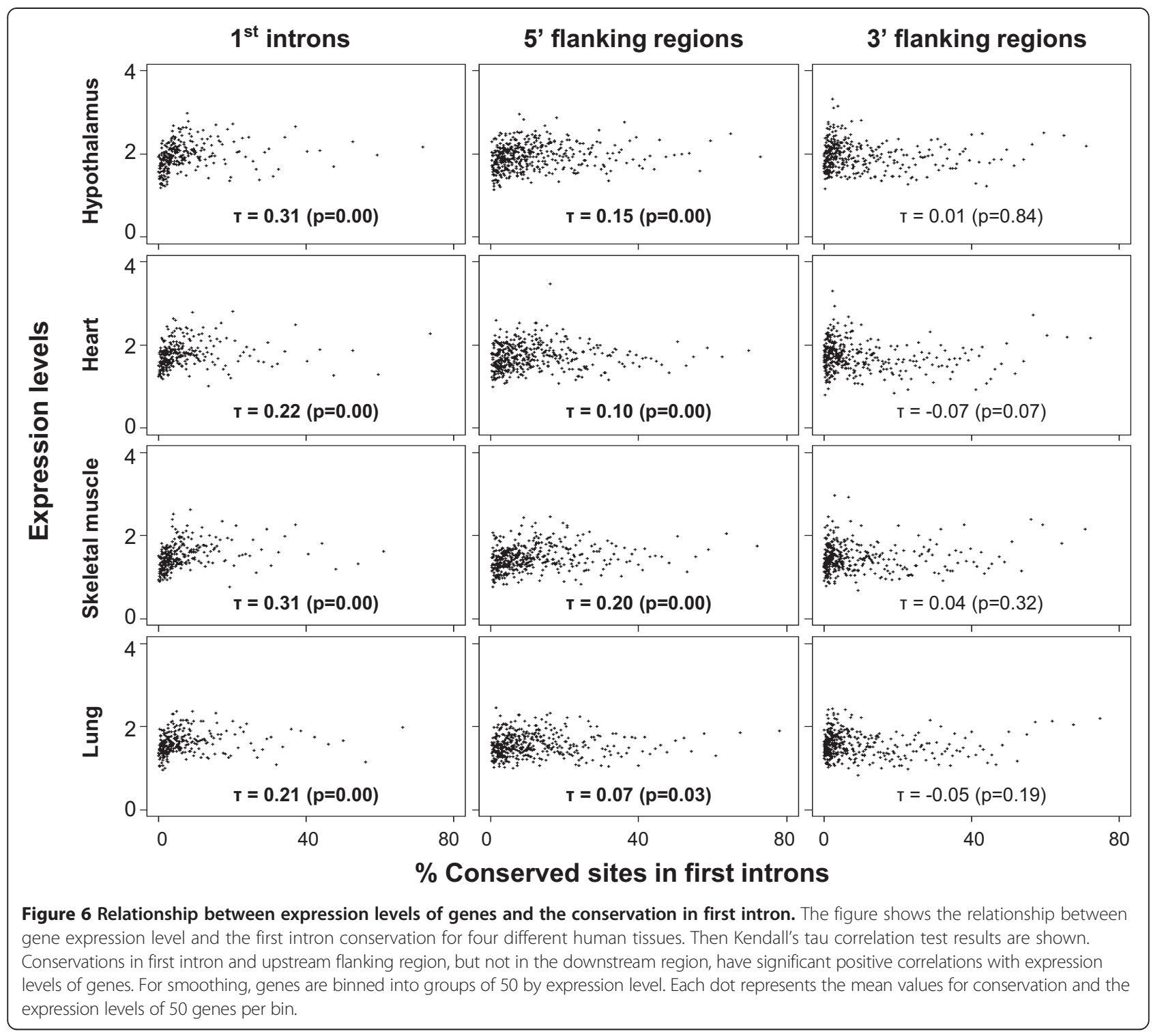

transcription start site, into the introns [56,64]. A 5' enrichment of certain epigenetic marks has also been noted with regards to splicing signals [35].

We first investigated whether the proportions of conservation and epigenetic marks are biased toward the 5' end of the intron. After excluding the short first introns (shorter than the median length) each intron was binned into five equal-sized bins, and we estimated for each bin, the fraction of introns in which the highest signal in the intron was in the particular bin. As shown in Additional file 1: Figure S9, there is varying enrichment of signals toward the 5' bins but in absolute terms only in a small fraction of introns the highest signal is in the 5'-most bin. Moreover, this trend is not uniformly observed for all regulatory signals.

Despite an enrichment of signals toward 5' as well as the fact that the general patterns of enrichment in the first introns also hold for the 5' flanking regions (Additional file 1: Figure S4 and S5), it is not immediately clear whether the patterns in the two regions are related by virtue of extended conserved and regulatory regions spanning the promoter and the first introns. To ensure that the observed patterns in first introns are not simply due to signals spanning the two regions, we tested if the trends in the first introns are maintained after removing such "spillover" signals. We reasoned that the genes in which a particular signal in first intron is simply due to spillover from the promoter should exhibit a greater proportion of that signal in promoter and first exon relative to the first intron. Thus we excluded the genes in which the proportion of a signal in the first exon and promoter was at least as high as that in the first intron. Despite the reduction in statistical power owing to much reduced dataset, all the trends were still maintained 


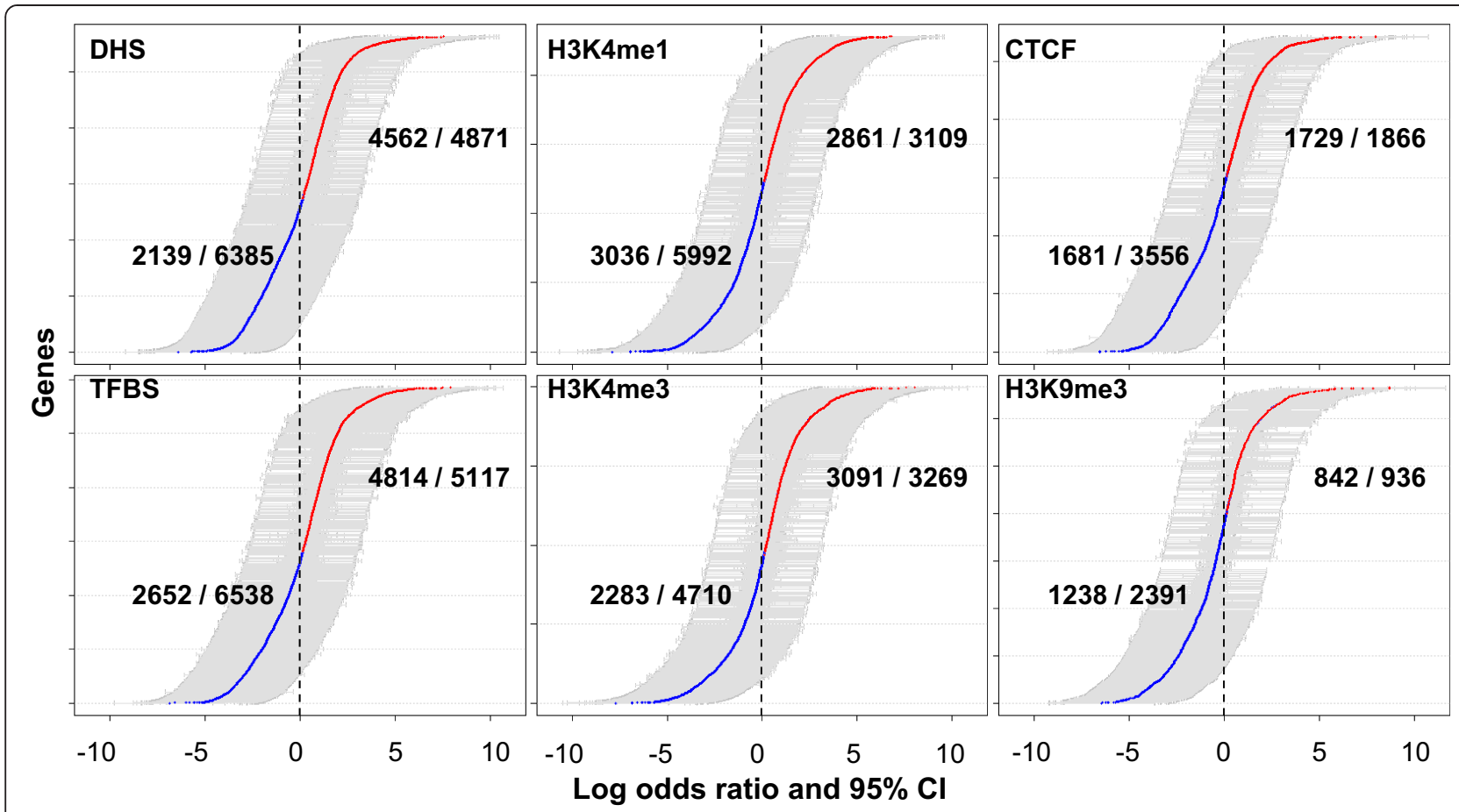

Figure 7 Enrichment of regulatory signals in conserved portion of first intron relative to non-conserved portion. After dividing each first intron into two groups, conserved sites and non-conserved sites, log-odds ratios ( $X$-axis) are computed with 95\% confidence interval (CI) (light gray bars) for each gene. The log-odds greater than zero are represented by red dots. Each box provides the analysis result done for each regulatory mark. $Y$ axis represents each gene corresponding to each log-odds ratio. The numbers of genes with a statistically significance $(p<0.01)$ divided by the total numbers of genes used for testing are presented in the middle of each box.

(Additional file 1: Figure S10A-D). Next, to exclude the interference of signals between introns and exons or flanking regions of other genes, we repeated the analyses after excluding the genes whose first intron overlapped with exon or flanks of another gene. As shown in Additional file 1: Figure S11A-D, all trends in the first introns are still maintained after removing the overlapped sets. All these results suggest that the first intron conservations and the enrichment of regulatory signals are independent of the trends for the promoter.

\section{Trends in the first introns are not due to their proximity} to the transcriptional start site

Given that many of the trends in first intron are similar to those in the proximal promoter, next we assessed whether the trends in the first introns are simply due to their proximity to the transcription start site (TSS). Thus we investigated the trends in the first introns compared to that in the second introns when controlled for their distance from the TSS. We categorized first and second introns into bins corresponding to distance of the 5 ' end of the intron from the TSS and compared the first and the second intron trends within each bin. Note that because first introns are much longer than the second intron (Additional file 1: Figure S12A) and because we are controlling for the distance to the 5' end of the intron, in the direct comparison, on average, the distances within first introns is much greater than those in second introns, thus rendering this comparison conservative. As shown in Additional file 1: Figure S12A, distances from the TSS to the first and the second intron are vastly different and overlap only in a short range $500-1000$ bps. For various distance bins within in this range, we compared the conservation and chromatin signal proportions between the first intron and the second intron.

As shown in Figure 8, the proportions of conservation, DHS, and TF sites in the first introns were almost always higher than in the second introns in all distance bins, suggesting that the observed trends are a property of first introns rather than of their proximity to the TSS alone. Furthermore, there was no clear difference between first and second introns for H3K4me1 and H3K4me3 signals, and there was no clear increasing or decreasing trends of conservation or signals with increasing the distances to the TSS (Figure 8). The loss of the trends in the two histone marks may partly be due to the breadth of those signals often spanning the entire genic regions, thus introducing error in estimation of precise proportions of those signals, which can overwhelm real signals in a relatively small dataset. Another possible explanation for the loss is cell-type dependency of those signals. As shown in Additional file 1: Figure S12B, the trend of H3K4me1 is 
(A)

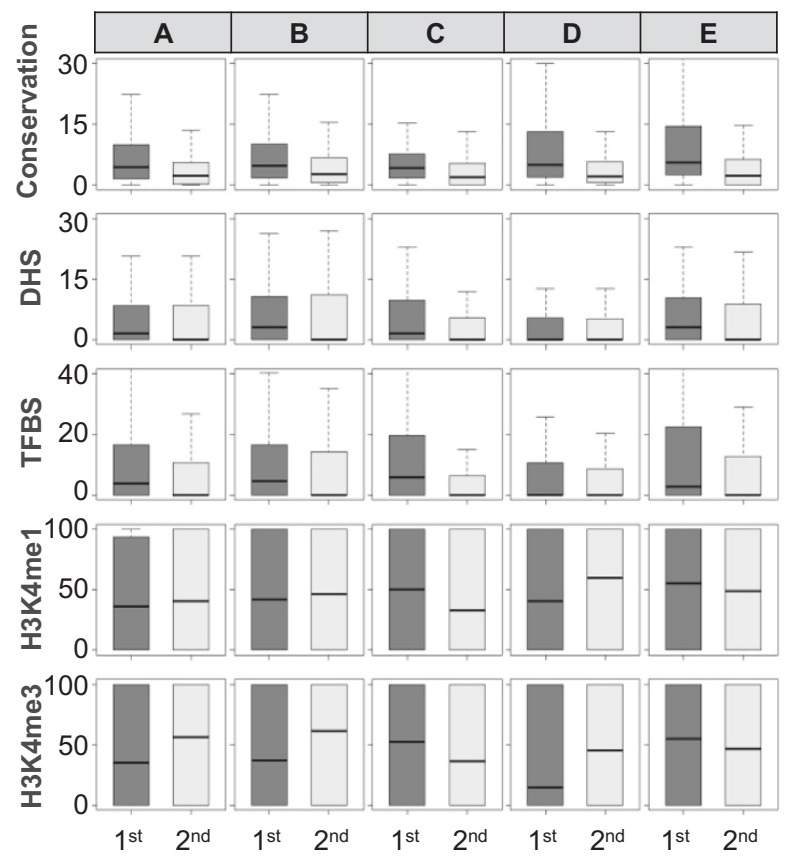

(B)

\begin{tabular}{|c|c|c|c|c|c|}
\cline { 2 - 6 } \multicolumn{1}{c|}{} & A & B & C & D & E \\
\hline $\begin{array}{c}\text { Range of distance } \\
\text { (bp) }\end{array}$ & $\begin{array}{c}500 \\
\sim 600\end{array}$ & $\begin{array}{c}600 \\
\sim 700\end{array}$ & $\begin{array}{c}700 \\
\sim 800\end{array}$ & $\begin{array}{c}800 \\
\sim 900\end{array}$ & $\begin{array}{c}900 \\
\sim 1000\end{array}$ \\
\hline $\begin{array}{c}\text { Number of } \\
\text { 1st introns }\end{array}$ & 895 & 482 & 269 & 177 & 120 \\
\hline $\begin{array}{c}\text { Number of } \\
\text { 2nd introns }\end{array}$ & 316 & 336 & 337 & 293 & 312 \\
\hline
\end{tabular}

One-sided Wilcoxon rank sum tests between $1^{\text {st }}$ introns and $2^{\text {nd }}$ introns in the same ranges of distance

\begin{tabular}{|c|c|c|c|c|c|c|}
\hline & Conservation & 0.00 & 0.00 & 0.00 & 0.00 & 0.00 \\
\hline & DHS & 0.00 & 0.00 & 0.00 & 0.19 & 0.01 \\
\hline & TFBS & 0.00 & 0.00 & 0.00 & 0.17 & 0.00 \\
\hline & H3K4me1 & 0.88 & 0.86 & 0.19 & 0.98 & 0.41 \\
\hline & H3K4me3 & 1.00 & 0.97 & 0.05 & 1.00 & 0.35 \\
\hline
\end{tabular}

Figure 8 Comparison of trend in first and second introns after controlling for their distance from the TSS. Using the start of first exon as a proxy for the TSS, distance from the first intron and the second intron to the TSS was obtained. Additional file 1: Figure S10A shows the length distribution of the two introns. Only the introns whose distance from TSS was in the overlapping range of 500-1000 bps were included in this analysis. Within this distance range, first and second introns were partitioned into smaller distance bins, and within each bin, various marks were compared between the first and the second introns. (A) Dark gray and light gray represent the proportions estimated in the first and the second intron respectively. (B) Table for the number of genes and corresponding statistics estimated by one-sided Wilcoxon rank sum tests for each comparison illustrated in (A).

still valid for h1 ESC cell line. Repetition of these analyses in other cell types (Additional file 1: Figure S12B, C) shows consistent results, indicating that the proximity of first introns to the TSS is not the main cause for the observed trends in the first introns.

\section{Discussion}

Introns are ultimately removed from protein synthesis as a part of post-transcriptional processing, yet all the intron sequences are respectively copied as pre-mRNAs or DNAs sequence-by-sequence during transcription and replication, which seems to cause huge energetic burden to cell. About 2 ATP is known to be consumed for $1 \mathrm{bp}$ synthesis during transcription [65]. Considering that introns can be as long as hundreds of kilobases, their maintenance must entail a substantial cost to the cells. Nevertheless, most eukaryotic genes have introns [1-6], and although introns can be lost, in general, introns have been maintained during eukaryotic lineage evolution $[5,6,66,67]$. Evolutionary maintenance of introns despite the energetic burden they entail suggests some evolutionary advantage afforded by introns.

Several studies have presented various possible scenarios for how introns provide advantage to cell's survival [68-72].
It is also true that, for a long time, introns have been considered to be non-essential for the most part [19-21]. In fact, approximately $3 \%$ of genes in the human genome are intronless genes [73]. Furthermore, no significant functional changes have been detected in many experimental designs with or without introns for the same coding sequences [74]; however this counterintuitive finding may be simply due to the fact that in molecular biology experiments, 'gene function' generally has been equated to 'protein function', devoid of its regulatory context.

Transcriptional regulatory signals encoded within introns represent one of the main selective advantages afforded by introns [32]. Two types of regulatory signals have been reported in introns; classical enhancers and intronmediated enhancers (IME). Several classical enhancers, i.e., cis-regulatory elements regulating spatio- temporal gene expression, are known to locate within introns in mouse transgenic experiments [75], for instance, the enhancer elements of GLI3, an important transcription factor of Sonic hedgehog signaling [76]. In contrast, IME suggest a broad role of an intron, first intron in particular, in regulating expression level, without ascribing the function to a specific region within the intron, suggesting a mechanism different from that for classical enhancers 
$[32,77]$. For instance, in experiments performed in Arabidopsis, rice, as well as mammal, the expression level of a gene with intron, particularly first intron, could increase up to 100-fold compared to the expression construct with the same coding sequences but without the introns [78]. IME activity was found to be dependent on the location and distance from transcription start site unlike the classical enhancers [79]. Whether or not IME is a general mechanism of expression control is not known.

Here, we have investigated the functional importance of first introns in human by quantifying their evolutionary conservation and potential regulatory content relative to other introns. Sequence conservation is considered to reflect the resistance against random mutations through purifying selection. Identifying conserved regions in genomes has thus been one of primary criterion to detect functional regions of genomes. Previous studies in several species including Drosophila and Arabidopsis [27,37,41,80] have shown that first introns tend to be the longest and the most conserved, which is recapitulated in human by our study (Figure 1). We further investigated the reasons of higher conservations in first introns by testing their association with various regulatory marks, and the associations of conservation and regulatory signals in first introns with the gene's expression level (Figures 2, 3, 4, 5 and 6). This analysis also underscores the importance of epigenomic data, which became available only recently by ENCODE project (Table 1), in interpreting the function of the non-coding portion of the genome. One of the interesting findings of this work is that genes with higher density of conservation and active regulatory marks but not repressive marks in first introns tend to have more exons that encode longer proteins (Figures 4 and 5), which can be interpreted to suggest that long functionally complex proteins may also be under a richer regulatory control. It is not entirely clear why only active regulatory signals but not repressive signals have positive correlations with conservation and number of exons. It may partly be due to the tendency of repressive signals to be broad and less intense relative to activating signals [64], which can result in lower discrimination of a specific region as well as lower detectability and lower statistical power. Overall, by leveraging the recent explosion in epigenomic data, our work lends further support, particularly in human, to the notion that introns, and especially the first introns, harbor evolutionarily constrained regulatory regions mediating both the level and complexity of gene expression. However several important questions remain open.

In our gene-specific analysis of enrichment of various regulatory signals in the conserved portion of the first intron, we found that in first introns, the conserved regions are favored by DHS, TFBS binding, and H3K4me3, which suggests that the conserved region may have a role in active gene regulation. More interestingly, we found a difference in two activating marks - H3K4me1 and H3K4me3. While both these marks are associated with proximal promoters, only H3K4me1 is associated with distal enhancers [54]. This subtle difference in enrichment may suggest that the conserved regions in the first intron are more promoter-like and less like a distal enhancer in their mode of action. However, this effect is not simply due to spillover of promoter signals into enhancer as we showed above (Additional file 1: Figure S10A-D). Further mechanistic disambiguation of this difference will require additional studies.

\section{Conclusions}

In the present study, we investigated the potential regulatory role of first introns in human genes by leveraging the recent explosion in epigenomic data by the ENCODE project. In addition to extending the previous results in Drosophila and plant to human, i.e. showing that the first introns are enriched for conserved regions, we show that these higher conservations in first introns are related to 1) the presence of active regulatory chromatin marks, 2) higher expression levels of genes, and 3) a greater number of exons within genes. Overall, our results strongly suggest that first introns in human are enriched for evolutionarily selected active transcriptional regulatory signals that are likely to be important for regulating complex gene expression patterns of large multi-domain genes.

The precise mechanism by which individual conserved, putative regulatory regions in the first intron, regulate gene expression, as well as other potential functions of conserved regions in the first intron, are unclear. The extreme lengths of mammalian first introns represent another enigma. The evolutionary path leading up to long introns as well as whether the first intron length is under selective constraint are not known. Finally, whether it is beneficial for the regulatory elements to reside within the first intron, as opposed to, say, the upstream region of the gene, or whether evolution is neutral to this outsideinside choice, is another open and interesting question. To ultimately resolve the mystery of introns, these are some of the questions that will need to be addressed.

\section{Methods}

Obtaining of introns, 5'-, and 3'-flanking regions from the human genome

The exon-intron position information of 36,024 Refseq mRNA (KnownGenes) without duplicates was downloaded from the UCSC Table Browser (January 2013). Genomic sequences for each chromosome were obtained from the primary GRCh37/hg19 assembly, and were used for retrieve intron sequences. Very short introns (less the $1 \mathrm{~kb}$ in length) were excluded, as well as very long introns (greater than (third quartile + (interquartile range $\times 1.5$ ) in length to minimize the outlier effects. To minimize 
interference from splicing signals in interpreting intronic conservation, we excluded 300 bps from both the 5' and 3' ends of each intron [42]. Then repeat elements were masked by RepeatMasker [81]. The numbers for introns in each ordinal position group after all these filtrations are shown in Figure 1. Additionally, we obtained the $2 \mathrm{~kb}$ region upstream of the first exon and $2 \mathrm{~kb}$ region downstream of the last exon, as a proxy for 5' and 3' flanks of genes.

\section{Estimating conservation}

PhastCons scores were used to estimate position-wise sequence conservation [82]. The PhastCons scores for 46 placental mammal subset were downloaded from the UCSC genome browser (Table 1, March 2013). The Phastcons scores were overlaid onto the intron regions and 5 '/3'-flanking regions obtained by the methods described above section. Sites with PhastCons score $\geq 0.5$ were considered as 'Conserved' sites. The proportions of conserved sites were then estimated for each group of introns grouped by their ordinal positions, and 5'/3'flanking regions. Conservation in first intron for each gene was estimated by the number of conserved sites divided by the total length of the intron.

\section{Obtaining and mapping regulatory signals and chromatin marks}

Genome positions of peaks (region of statistically significant signal enrichment) for DHSs, TFBSs and chromatin marks measured in the ENCODE Tier-1 cell lines (GM12878, H1-hESC and K562) were downloaded from the ENCODE Project from UCSC genome browser (March 2013). The specific download links are provided in Table 1. The processed peak regions of all of these regulatory signals were extracted, and mapped onto the filtered introns and flanking regions. For each intron and flanking region, we then estimated the proportion of the region covered by each regulatory signal and chromatin mark.

\section{Obtaining mRNA expression levels estimated by RNA-seq analysis}

Krupp et al. [63] have reported the mRNA expression levels in RPKM. We used $\log _{2}(\mathrm{RPKM}+1)$ to report expression levels of mRNAs for 11 different human tissues (Table 1). A total of 32,384 mRNAs have their expression values.

\section{Statistical tests}

Kendall's tau rank correlation coefficients were computed using R studio (Racine 2012) among the variables representing evolutionary conservations of introns or flanking regions, regulatory signals mapped onto introns or flanking regions, and mRNA expression levels. Introns or flanking regions with no conservation or regulatory signal were excluded; we ascertained that this only makes our results more stringent because the fraction of first introns that were eliminated were smaller than those for other introns and including this with " 0 " value would make the results even stronger.

To test whether the regulatory signals and chromatin marks are enriched in conserved regions compared to non-conserved regions in each gene, odds ratio of the signal estimates between the two regions was estimated. Briefly, first introns were divided into two regions, conserved first introns and non-conserved first introns by the criteria of Phastcons $\geq 0.5$, and the chromatin signals and regulatory signals described in the main text were overlaid into each region, generating a $2 \times 2$ contingency table. Odds ratios were then estimated for each gene from the contingency table. The odds ratio is the ratio of the proportion of regulatory signals in conserved sequences to that in non-conserved sequences. The odds ratios were thus computed using the "Text::NSP::Neasures::2D::odds" Perl module, and the 95\% confidence intervals associated with odds ratios were calculated using the formulas, 'In (odds ratio $) \pm 1.96 \times \sqrt{\frac{1}{R C}+\frac{1}{N C}+\frac{1}{R N}+\frac{1}{N N}}$, (SC: Signal peaks on conserved sites, NC: Non-signals peaks on conserved sites, SN: Signal peaks on non-conserved sites, NN: Non-signal peaks on non-conserved sites) [83].

\section{Additional file}

Additional file 1: Figure S1. Comparison of conservations in first introns with those in the other introns using an alternative grouping strategy. Figure S2. Proportions of regulatory chromatin marks in intron ordinal groups in $\mathrm{H} 1$-hESC and K562. Figure S3. Correlation between regulatory signals and conservation in first introns in $\mathrm{H} 1-\mathrm{hESC}$ and $\mathrm{K} 562$. Figure S4. Correlation between regulatory signals and conservation in the upstream flanking regions in three different cell lines. Figure S5. Relationship between flanking region conservation and the numbers of exons. Figure S6. Relationship between the proportions of regulatory signals in introns of each ordinal position and the numbers of exons. Figure S7. Analysis based on a single representative transcript for each gene. Figure S8. Enrichment of regulatory marks in the first intron in two additional cell lines. Figure S9. Five prime to three prime biases in signal density along the first intron. Figure S10. Excluding spillover of signals s from the promoter. Figure S11. Excluding genes whose first introns overlapped with exons or flanks of another genes. Figure S12. Analyzing the effect of proximity to the TSS.

\section{Competing interests}

The authors declare that they have no competing interests.

\section{Authors' contributions}

$\mathrm{SH}$ and SSC conceived the project, designed the research plan, and wrote the manuscript. SGP performed the analysis. All authors have read and approved the final manuscript.

\section{Acknowledgements}

This research was supported by Basic Science Research Program through the National Research Foundation of Korea (NRF) funded by the Ministry of Education, Science and Technology (NRF-2014R1A1A4A01003793) and by a grant of the Korean Health Technology R\&D Project, Ministry of Health \& Welfare, Republic of Korea (HI13C0016) to S.S.C, and by NIH grant GM100335 to S.H. Authors would like to thank Steve Mount, Srutii Sarda, and Avinash Das for their useful comments. 
Received: 7 January 2014 Accepted: 18 June 2014

Published: 26 June 2014

\section{References}

1. Berget SM, Moore C, Sharp PA: Spliced segments at the 5'terminus of adenovirus 2 late mRNA. Proc Natl Acad Sci 1977, 74(8):3171-3175.

2. Chow LT, Gelinas RE, Broker TR, Roberts RJ: An amazing sequence arrangement at the $5^{\prime}$ ends of adenovirus 2 messenger RNA. Cell 1977, 12(1):1-8.

3. Hawkins JD: A survey on intron and exon lengths. Nucleic Acids Res 1988, 16(21):9893-9908

4. Deutsch M, Long M: Intron-exon structures of eukaryotic model organisms. Nucleic Acids Res 1999, 27(15):3219-3228.

5. Simpson AG, MacQuarrie EK, Roger AJ: Eukaryotic evolution: early origin of canonical introns. Nature 2002, 419(6904):270.

6. Koonin EV: The origin of introns and their role in eukaryogenesis: a compromise solution to the introns-early versus introns-late debate? Biol Direct 2006, 1(1):22.

7. Wu J, Xiao J, Wang L, Zhong J, Yin H, Wu S, Zhang Z, Yu J: Systematic analysis of intron size and abundance parameters in diverse lineages. SCi China Life Sci 2013, 56(10):968-974.

8. Nixon JE, Wang A, Morrison HG, McArthur AG, Sogin ML, Loftus BJ, Samuelson J: A spliceosomal intron in Giardia lamblia. Proc Natl Acad Sci U S A 2002, 99(6):3701-3705.

9. Berget SM: Exon recognition in vertebrate splicing. J Biol Chem 1995, 270(6):2411-2414

10. Logsdon JM Jr: The recent origins of spliceosomal introns revisited. Curr Opin Genet Dev 1998, 8(6):637-648.

11. Lynch M: Intron evolution as a population-genetic process. Proc Natl Acad Sci 2002, 99(9):6118-6123.

12. Mourier T, Jeffares DC: Eukaryotic intron loss. Science 2003, 300(5624):1393.

13. Jeffares DC, Mourier T, Penny D: The biology of intron gain and loss. Trends Genet 2006, 22(1):16-22.

14. Lynch M, Conery JS: The origins of genome complexity. Science 2003 , 302(5649):1401-1404.

15. Gregory TR: Synergy between sequence and size in large-scale genomics. Nat Rev Genet 2005, 6(9):699-708.

16. Taft RJ, Pheasant M, Mattick JS: The relationship between non-protein-coding DNA and eukaryotic complexity. Bioessays 2007, 29(3):288-299.

17. Rogozin IB, Carmel L, Csuros M, Koonin EV: Origin and evolution of spliceosomal introns. Biol Direct 2012, 7:11-6150-7-11.

18. Sakurai A, Fujimori S, Kochiwa H, Kitamura-Abe S, Washio T, Saito R, Carninci $P$, Hayashizaki $Y$, Tomita M: On biased distribution of introns in various eukaryotes. Gene 2002, 300(1):89-95.

19. Gilbert W: Why genes in pieces? Nature 1978, 271(5645):501.

20. Li W, Graur D: Fundamentals of Molecular Evolution. Sunderland, MA: Sinauer Associates; 1991

21. Li W: Molecular Evolution. Sunderland, MA: Sinauer Associates Incorporated; 1997.

22. Halligan DL, Eyre-Walker A, Andolfatto P, Keightley PD: Patterns of evolutionary constraints in intronic and intergenic DNA of Drosophila. Genome Res 2004, 14(2):273-279.

23. Jareborg N, Birney E, Durbin R: Comparative analysis of noncoding regions of 77 orthologous mouse and human gene pairs. Genome Res 1999, 9(9):815-824.

24. Shabalina SA, Kondrashov AS: Pattern of selective constraint in C. elegans and C. briggsae genomes. Genet Res 1999, 74(1):23-30.

25. Bergman CM, Kreitman M: Analysis of conserved noncoding DNA in Drosophila reveals similar constraints in intergenic and intronic sequences. Genome Res 2001, 11(8):1335-1345.

26. Sironi M, Menozzi G, Comi GP, Bresolin N, Cagliani R, Pozzoli U: Fixation of conserved sequences shapes human intron size and influences transposon-insertion dynamics. Trends Genet 2005, 21(9):484-488.

27. Caenorhabditis elegans Sequencing Consortium: Genome sequence of the nematode C. elegans: a platform for investigating biology. Science 1998, 282(2012):2018.

28. Vinogradov AE: "Genome design" model: Evidence from conserved intronic sequence in human-mouse comparison. Genome Res 2006, 16(3):347-354

29. Haddrill PR, Charlesworth B, Halligan DL, Andolfatto P: Patterns of intron sequence evolution in Drosophila are dependent upon length and GC content. Genome Biol 2005, 6(8):R67.
30. Ladd AN, Cooper TA: Finding signals that regulate alternative splicing in the post-genomic era. Genome Biol 2002, 3(11):1-16.

31. Cartegni L, Chew SL, Krainer AR: Listening to silence and understanding nonsense: exonic mutations that affect splicing. Nat Rev Genet 2002, 3(4):285-298.

32. Rose A: Intron-mediated regulation of gene expression. Curr Top Microbiol Immunol 2008, 326:277-290.

33. Nott A, Meislin SH, Moore MJ: A quantitative analysis of intron effects on mammalian gene expression. RNA 2003, 9(5):607-617.

34. Tilgner H, Knowles DG, Johnson R, Davis CA, Chakrabortty S, Djebali S, Curado J, Snyder M, Gingeras TR, Guigo R: Deep sequencing of subcellular RNA fractions shows splicing to be predominantly co-transcriptional in the human genome but inefficient for IncRNAs. Genome Res 2012, 22(9):1616-1625

35. Bieberstein NI, Carrillo Oesterreich F, Straube K, Neugebauer KM: First exon length controls active chromatin signatures and transcription. Cell Rep 2012, 2(1):62-68.

36. Keightley PD, Gaffney DJ: Functional constraints and frequency of deleterious mutations in noncoding DNA of rodents. Proc Natl Acad Sci 2003, 100(23):13402-13406

37. Marais G, Nouvellet P, Keightley PD, Charlesworth B: Intron size and exon evolution in Drosophila. Genetics 2005, 170(1):481-485.

38. Zheng Y, Dimond KL, Graur D, Zufall RA: Patterns of intron sequence conservation in the genus Tetrahymena. Protist Genomics 2013, 1:19-24.

39. Majewski J, Ott J: Distribution and characterization of regulatory elements in the human genome. Genome Res 2002, 12(12):1827-1836.

40. Gaffney DJ, Keightley PD: Unexpected conserved non-coding DNA blocks in mammals. Trends Genet 2004, 20(8):332-337.

41. Bradnam KR, Korf I: Longer first introns are a general property of eukaryotic gene structure. PLoS One 2008, 3(8):e3093.

42. Barash Y, Calarco JA, Gao W, Pan Q, Wang X, Shai O, Blencowe BJ, Frey BJ: Deciphering the splicing code. Nature 2010, 465(7294):53-59.

43. Emorine $L$, Kuehl $M$, Weir $L$, Leder $P, M a x E E: A$ conserved sequence in the immunoglobulin JK-CK intron: possible enhancer element. ; 1983.

44. Lou H, Yang Y, Cote GJ, Berget SM, Gagel RF: An intron enhancer containing a 5 'splice site sequence in the human calcitonin/ calcitonin gene-related peptide gene. Mol Cell Biol 1995, 15(12):7135-7142.

45. Haerry TE, Gehring WJ: Intron of the mouse Hoxa-7 gene contains conserved homeodomain binding sites that can functionas an enhancer element in Drosophila. Proc Natl Acad Sci 1996, 93(24):13884-13889.

46. Keegan LP, Haerry TE, Crotty DA, Packer Al, Wolgemuth DJ, Gehring WJ: A sequence conserved in vertebrate Hox gene introns functions as an enhancer regulated by posterior homeotic genes in Drosophila imaginal discs. Mech Dev 1997, 63(2):145-157.

47. Surinya $\mathrm{KH}, \mathrm{Cox} \mathrm{TC}$, May BK: Identification and characterization of a conserved erythroid-specific enhancer located in intron 8 of the human 5-aminolevulinate synthase 2 gene. J Biol Chem 1998, 273(27):16798-16809.

48. Feng W, Huang J, Zhang J, Williams T: Identification and analysis of a conserved Tcfap2a intronic enhancer element required for expression in facial and limb bud mesenchyme. Mol Cell Biol 2008, 28(1):315-325.

49. Visel A, Rubin EM, Pennacchio LA: Genomic views of distant-acting enhancers. Nature 2009, 461(7261):199-205.

50. Sauter KA, Bouhlel MA, O'Neal J, Sester DP, Tagoh H, Ingram RM, Pridans C, Bonifer C, Hume DA: The Function of the Conserved Regulatory Element within the Second Intron of the Mammalian Csf1r Locus. PLoS One 2013, 8(1):e54935.

51. Hsu AP, Johnson KD, Falcone EL, Sanalkumar R, Sanchez L, Hickstein DD, Cuellar-Rodriguez J, Lemieux JE, Zerbe CS, Bresnick EH: GATA2 haploinsufficiency caused by mutations in a conserved intronic element leads to MonoMAC syndrome. Blood 2013, 121(19):3830-3837.

52. Martin $C$, Zhang $Y$ : The diverse functions of histone lysine methylation. Nat Rev Mol Cell Biol 2005, 6(11):838-849.

53. Kouzarides T: Chromatin modifications and their function. Cell 2007 128(4):693-705.

54. Ernst J, Kellis M: ChromHMM: automating chromatin-state discovery and characterization. Nat Methods 2012, 9(3):215-216.

55. ENCODE Project Consortium: A user's guide to the encyclopedia of DNA elements (ENCODE). PLoS Biol 2011, 9(4):e1001046. 
56. ENCODE Project Consortium, Bernstein BE, Birney E, Dunham I, Green ED, Gunter C, Snyder M: An integrated encyclopedia of DNA elements in the human genome. Nature 2012, 489(7414):57-74.

57. Yuan $H$, Wang $Y$, Wu $X$, Wang $H$, Pu J, Ding W, Wang $M$, Shen $X$, Cong $H$, Zhang $L$ : Characterization of the 5 '-flanking region and regulation of transcription of human BAFF-R gene. DNA Cell Biol 2010, 29(3):133-139.

58. Venkataraman GM, Suciu D, Groh V, Boss JM, Spies T: Promoter region architecture and transcriptional regulation of the genes for the $\mathrm{MHC}$ class I-related chain A and B ligands of NKG2D.J Immunol 2007, 178(2):961-969.

59. Rippe R, Lorenzen S, Brenner D, Breindl M: Regulatory elements in the 5 '-flanking region and the first intron contribute to transcriptional control of the mouse alpha 1 type I collagen gene. Mol Cell Biol 1989, 9(5):2224-2227.

60. O'Carroll A, Lolait SJ, Howell GM: Transcriptional regulation of the rat apelin receptor gene: promoter cloning and identification of an Sp1 site necessary for promoter activity. J Mol Endocrinol 2006, 36(1):221-235.

61. Donaldson ZR, Young LJ: The Relative Contribution of Proximal 5' Flanking Sequence and Microsatellite Variation on Brain Vasopressin 1a Receptor (Avpr1a) Gene Expression and Behavior. PLoS Genet 2013, 9(8):e1003729.

62. Vinogradov AE: 'Genome design'model and multicellular complexity: golden middle. Nucleic Acids Res 2006, 34(20):5906-5914

63. Krupp M, Marquardt JU, Sahin U, Galle PR, Castle J, Teufel A: RNA-Seq Atlas - $a$ reference database for gene expression profiling in normal tissue by next-generation sequencing. Bioinformatics 2012, 28(8):1184-1185.

64. Barth TK, Imhof A: Fast signals and slow marks: the dynamics of histone modifications. Trends Biochem Sci 2010, 35(11):618-626.

65. Alberts B: Molecular Biology of the Cell. New York: Garland Science; 2000.

66. Palmer JD, Logsdon JM Jr: The recent origins of introns. Curr Opin Genet Dev 1991, 1(4):470-477

67. Rodríguez-Trelles F, Tarrío R, Ayala FJ: Origins and evolution of spliceosomal introns. Annu Rev Genet 2006, 40:47-76.

68. Duret $L$ : Why do genes have introns? Recombination might add a new piece to the puzzle. Trends Genet 2001, 17(4):172-175.

69. Fedorova L, Fedorov A: Introns in gene evolution. Genetica 2003, $118(2-3): 123-131$

70. Niu DK: Protecting exons from deleterious R-loops: a potential advantage of having introns. Biol Direct 2007, 2:11.

71. Fedorova L, Fedorov A: Puzzles of the human genome: Why do we need our introns? Curr Genomics 2005, 6(8):589-595.

72. Fedorov A, Fedorova L: Introns: mighty elements from the RNA world. J Mol Evol 2004, 59(5):718-721.

73. Grzybowska EA: Human intronless genes: functional groups, associated diseases, evolution, and mRNA processing in absence of splicing. Biochem Biophys Res Commun 2012, 424(1):1-6.

74. Parenteau J, Durand M, Véronneau S, Lacombe A, Morin G, Guérin V, Cecez B, Gervais-Bird J, Koh C, Brunelle D: Deletion of many yeast introns reveals a minority of genes that require splicing for function. Mol Biol Cell 2008, 19(5):1932-1941

75. Oswald A, Oates AC: Control of endogenous gene expression timing by introns. Genome Biol 2011, 12(3):107-2011-12-3-107.

76. Abbasi AA, Paparidis Z, Malik S, Bangs F, Schmidt A, Koch S, Lopez-Rios J, Grzeschik K: Human intronic enhancers control distinct sub-domains of Gli3 expression during mouse CNS and limb development. BMC Dev Biol 2010, 10(1):44

77. Jeong Y, Mun J, Lee I, Woo JC, Hong CB, Kim S: Distinct roles of the first introns on the expression of Arabidopsis profilin gene family members. Plant Physiol 2006, 140(1):196-209.

78. Callis J, Fromm M, Walbot V: Introns increase gene expression in cultured maize cells. Genes Dev 1987, 1(10):1183-1200.

79. Parra G, Bradnam K, Rose AB, Korf I: Comparative and functional analysis of intron-mediated enhancement signals reveals conserved features among plants. Nucleic Acids Res 2011, 39(13):5328-5337.

80. Chung B, Simons C, Firth A, Brown C, Hellens R: Effect of 5'UTR introns on gene expression in Arabidopsis thaliana. BMC Genomics 2006, 7(1):120.

81. Smit AF, Hubley R, Green P: RepeatMasker Open-3.0. 1996.
82. Siepel A, Bejerano G, Pedersen JS, Hinrichs AS, Hou M, Rosenbloom K, Clawson H, Spieth J, Hillier LW, Richards S: Evolutionarily conserved elements in vertebrate, insect, worm, and yeast genomes. Genome Res 2005, 15(8):1034-1050.

83. Morris JA, Gardner MJ: Statistics in Medicine: Calculating confidence intervals for relative risks (odds ratios) and standardised ratios and rates. Br Med J (Clin Res Ed) 1988, 296(6632):1313.

doi:10.1186/1471-2164-15-526

Cite this article as: Park et al: Conservation in first introns is positively associated with the number of exons within genes and the presence of regulatory epigenetic signals. BMC Genomics 2014 15:526.

\section{Submit your next manuscript to BioMed Central and take full advantage of:}

- Convenient online submission

- Thorough peer review

- No space constraints or color figure charges

- Immediate publication on acceptance

- Inclusion in PubMed, CAS, Scopus and Google Scholar

- Research which is freely available for redistribution

Submit your manuscript at www.biomedcentral.com/submit
C) Biomed Central 